\title{
A new beam emission polarimetry diagnostic for measuring the magnetic field line angle at the plasma edge of ASDEX Upgrade
}

\author{
E. Viezzer, ${ }^{1,2, a)}$ R. Dux, ${ }^{1}$ M. G. Dunne, ${ }^{1}$ and ASDEX Upgrade Team ${ }^{1, b)}$ \\ ${ }^{1}$ Max-Planck-Institute for Plasma Physics, Boltzmannstr. 2, 85748 Garching, Germany \\ ${ }^{2}$ Department of Atomic, Molecular, and Nuclear Physics, University of Seville, \\ Avda. Reina Mercedes, 41012 Seville, Spain
}

(Presented 8 June 2016; received 2 June 2016; accepted 3 July 2016; published online 18 August 2016)

\begin{abstract}
A new edge beam emission polarimetry diagnostic dedicated to the measurement of the magnetic field line angle has been installed on the ASDEX Upgrade tokamak. The new diagnostic relies on the motional Stark effect and is based on the simultaneous measurement of the polarization direction of the linearly polarized $\pi$ (parallel to the electric field) and $\sigma$ (perpendicular to the electric field) lines of the Balmer line $\mathrm{D}_{\alpha}$. The technical properties of the system are described. The calibration procedures are discussed and first measurements are presented. [http://dx.doi.org/10.1063/1.4959952]
\end{abstract}

\section{INTRODUCTION}

Accurate reconstructions of the internal magnetic field are important for understanding the stability of the pedestal and the occurrence of edge localized modes (ELMs). ELMs are magnetohydrodynamic instabilities which expel particles and energy from the edge of high confinement (H-mode) plasmas. They occur after the edge profiles steepen up to a limit that is thought to be defined by the peeling-ballooning limit, ${ }^{1}$ i.e., a maximum pressure gradient $(\nabla \mathrm{p})$ is reached, followed by a limit in the edge current density $(j)$.

Precise measurements of $j$ are difficult. The most common tool to diagnose $j$ is the Motional Stark Effect (MSE) system. It relies on the MSE from the Lorentzian electric field induced in the rest frame of the atom as the neutral beam propagates across a magnetic field. The Stark effect provokes wavelength splitting and polarization of the emitted radiation. Simultaneous spectral and polarization measurements of the direction of the linearly polarized $\pi$ (parallel to the electric field) and $\sigma$ (perpendicular to the electric field) component emission allow for a direct measurement of the magnetic field line angle $\gamma=\arctan \left(\mathrm{B}_{\theta} / \mathrm{B}_{\phi}\right)$, where $\mathrm{B}_{\theta}$ and $\mathrm{B}_{\phi}$ denote the poloidal and toroidal magnetic field. The edge current density is derived from $\gamma$ through $\mathrm{B}_{\theta}$ using Ampère's law. A change in $j$ results in a small change in $\gamma$ as it has to compete with the total plasma current. In addition, the existence of a strong radial electric field, $E_{r}$, in the edge pedestal poses a challenge on the measurement of $j$ since it will add to the induced Lorentzian electric field.

A new diagnostic for measuring the magnetic field line angle at the plasma edge has been installed at ASDEX Upgrade (AUG). The beam emission polarimetry (BEP) diagnostic is

Note: Contributed paper, published as part of the Proceedings of the $21 \mathrm{st}$ Topical Conference on High-Temperature Plasma Diagnostics, Madison, Wisconsin, USA, June 2016.

a) Author to whom correspondence should be addressed. Electronic addresses: eleonora.viezzer@ipp.mpg.de and eviezzer@us.es

b) For authors' list, see H. Zohm et al., Nucl. Fusion 55, 104010 (2015). an alternative approach based on the MSE. The polarized emission is measured simultaneously at different values of the polarization angle (here, $0^{\circ}, 45^{\circ}$, and $90^{\circ}$ ). This enables the measurement of the $\pi$ and $\sigma$ emissions at the same time and thus the reconstruction of $\gamma$ from the full MSE spectrum. In this paper, a system overview and first measurements from the new diagnostic on AUG are presented.

\section{DIAGNOSTIC SETUP AND HARDWARE SPECIFICATIONS}

The beam emission polarimetry diagnostic measures the Stark split emission from the Balmer line $D_{\alpha}(n=3 \rightarrow 2$ transition, $\lambda=656.1 \mathrm{~nm}$ ) of deuterium atoms from one of the neutral heating beams on AUG. The Stark splitting of the energy levels results from two contributions: (i) the Lorentzian electric field produced when the beam particles move across the magnetic field and (ii) the background radial electric field $E_{r}$. Hence, the total electric field is $\mathbf{E}=\mathbf{v}_{b} \times \mathbf{B}+\mathbf{E}_{r}$, where $\mathbf{v}_{b}$ is the velocity of the neutral beam particles and $\mathbf{B}$ the magnetic field. At AUG, the beam induced electric field is of the orders of $6 \mathrm{MV} / \mathrm{m}$ (for the full energy component of a $93 \mathrm{keV} \mathrm{D}$ beam at $2 \mathrm{~T}$ ) while the typical local radial electric field in the $\mathrm{H}$-mode transport barrier is $\sim 40-50 \mathrm{kV} / \mathrm{m} .{ }^{2}$ Despite being two orders of magnitude smaller, the inclusion of the local $E_{r}$ is important as it can make a significant contribution to the line ratios of the different optical heads. The signal change for an $E_{r}$ of $50 \mathrm{kV} / \mathrm{m}$ can account for up to $2.5 \%$ depending on the beam energy component, which is of the same order as the expected signal variation due to an ELM. In the pedestal, a change of the field line angle of $1^{\circ}$ corresponds to a variation of $\sim 2 \%$ in the measured signal.

The deuterium atoms show a linear Stark effect and the $\mathrm{D}_{\alpha}$ emission line splits into 15 lines, 9 strong and 6 very weak lines. The Stark lines are polarized perpendicular to the electric field (three $\sigma$ lines for $\Delta m= \pm 1$, with $m$ being the magnetic quantum number) and polarized parallel to the electric field (six $\pi$ lines for $\Delta m=0$ ). The measured radiance 
(a)

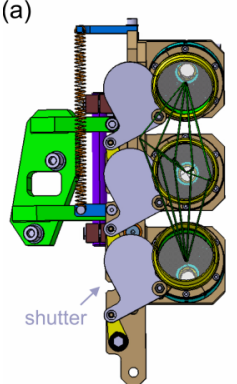

(b)

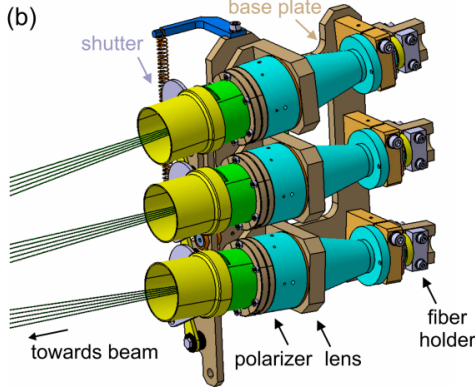

(c)

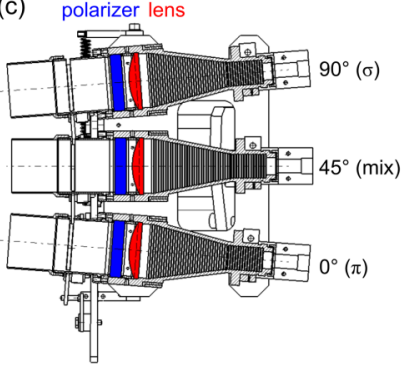

(d)

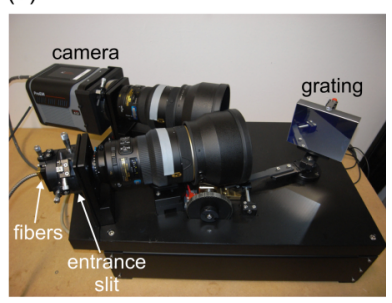

FIG. 1. Computer aided design (CAD) drawings of beam emission polarimetry diagnostic: (a) front view, (b) side view, (c) cut-through showing the position of the polarizers and the lenses. (d) Setup of lens-based spectrometer.

after a linear polarizer, which has an angle $\alpha$ with respect to the electric field, is proportional to $\sin ^{2}(\alpha)$ for the $\sigma$ lines and proportional to $\cos ^{2}(\alpha)$ for the $\pi$ lines. We use three independent observations of the same spot with polarizer angles $\alpha \approx 0^{\circ}, 45^{\circ}$, and $90^{\circ}$ (see Figure 1 ), where $0^{\circ}$ serves as a reference for the $\pi$ lines, $90^{\circ}$ for the $\sigma$ lines, and $45^{\circ}$ measures a combination of both $\sigma$ and $\pi$ lines.

Around the separatrix, the upper states of the $\mathrm{D}_{\alpha}$ transition are not necessarily in statistical equilibrium ${ }^{3}$ and the line ratio depends on the plasma density. ${ }^{4,5}$ Thus, the magnetic field line angle depends on the polarization fraction. Deviations from the values obtained assuming the Boltzmann statistical distribution of populations ${ }^{6}$ affect the line ratios between $\sigma$ lines or between $\pi$ lines and are visible in the measured spectra. The non-statistical equilibrium is taken into account in the forward model with non-Boltzmann occupation numbers of the upper levels (see Section IV).

Figure 1 shows the components of the beam emission polarimetry diagnostic, (a) a front view, (b) a side view, and (c) a cut-through of the optical setup, and (d) the spectrometer. The diagnostic uses three optical heads mounted at sector 6 inside the vessel. Each optical head is equipped with five high-temperature fibers with a diameter of $400 \mu \mathrm{m}$ which are focused on the center of the neutral beam source \#6, which is a $93 \mathrm{keV}$ deuterium beam of neutral beam injection (NBI) box 2 mounted in sector 7 of AUG, injected $\sim 30 \mathrm{~cm}$ below the midplane (see Figure 2(a)). The three optical heads view the same five radial locations at the focal point. The system features three $f / 2$ aspherical lenses (diameter $50 \mathrm{~mm}$, focal length $100 \mathrm{~mm}$ ) with an anti-reflexion coating for the visible range. Each optical head is equipped with a polarizer, mounted in front of the lens (see Figure 1(c)). The polarizers consist of $1.2 \mathrm{~mm}$ glass plates with implanted silver ellipsoids (filter thickness $250 \mu \mathrm{m}$ ). Each plate is laminated onto a $\mathrm{MgF}_{2}$ plate, which has a thickness of $1 \mathrm{~cm}$, in order to increase the heat capacity and to overcome the high power fluxes during a plasma discharge. The spot size in the focal plane is $4 \mathrm{~mm}$, while two adjacent lines of sight (LOS) are separated radially by $\sim 2 \mathrm{~cm}$, covering $\sim 10 \mathrm{~cm}$ of the edge plasma.

The interior of the optical heads is grooved with a sawtooth profile which acts like a beam dump to avoid reflections inside the optical head (see Figure 1(c)). The diagnostic also features a mechanical shutter (highlighted in grey in Figures 1(a) and 1(b)) to avoid impurity layers on the polarizers during boronizations, which are regularly performed on ASDEX Upgrade. For dedicated experiments, the shutter is opened well in advance.

The light collected along the LOS is transmitted to a high-throughput $f / 2$ Czerny-Turner like spectrometer, which has a variable entrance slit that is usually set to $50 \mu \mathrm{m}$. It utilizes two objective lenses (Nikon) with a focal length of $200 \mathrm{~mm}$ and a movable grating with 2400 grooves/mm (see Figure 1(d)). This choice of lenses enables the light from 16 fibers, which are stacked vertically along the entrance slit, to be imaged concurrently on the camera. One of these fibers is attached to a neon lamp which provides a shotto-shot wavelength calibration using the $\mathrm{Ne}$ spectral line at $659.9 \mathrm{~nm}$. The spectrometer features a new Princeton Instruments ProEM, back-illuminated, frame-transfer charged couple device (CCD) camera with on-chip multiplication gain and a $512 \times 51216 \mu \mathrm{m}$ pixel chip. At a central wavelength of $660 \mathrm{~nm}$, the dispersion of the spectrometer is $0.116 \AA /$ pixel.

Figure 2 shows an overview of the viewing geometry of the new diagnostic. In (a) a poloidal cross section and (b) a top-down view of AUG along with the LOS of the new diagnostic and the path of the neutral beam is illustrated.
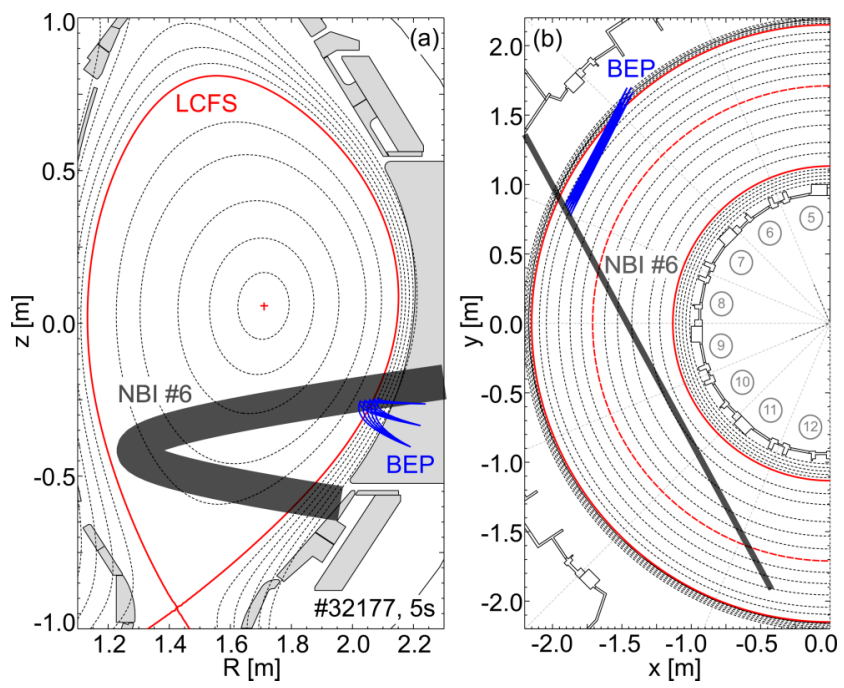

FIG. 2. (a) Poloidal cross section of AUG indicating the measurement locations of the beam emission polarimetry (BEP) diagnostic and (b) top-down view showing the lines of sight of BEP. The indicated beam lines do not correspond to the actual width of the beam $(\sim 20 \mathrm{~cm})$. 

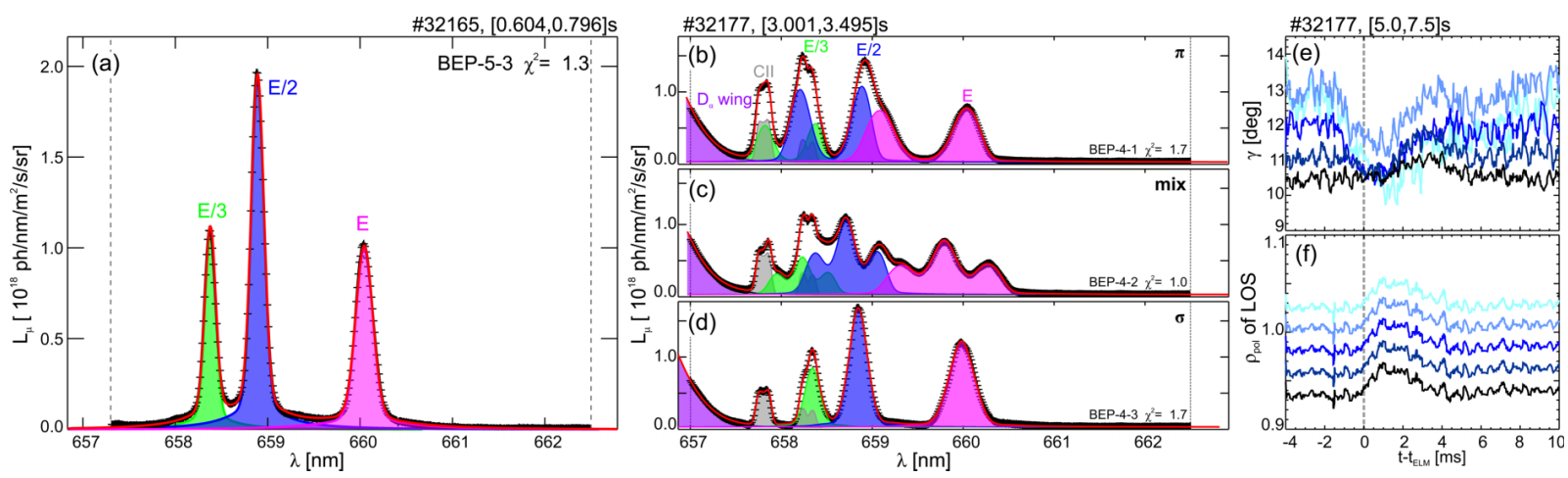

FIG. 3. (a) Beam into gas spectrum. Stark split $\mathrm{D}_{\alpha}$ spectrum of (b) $\pi$, (c) mixed and (d) $\sigma$ channel, (e) temporal evolution of the magnetic field line angle during the ELM cycle, (f) corresponding normalized poloidal flux label $\left(\rho_{p o l}\right)$ of each LOS.

\section{CALIBRATION}

Various alignment and calibration procedures are carried out in order to characterize the instrument and the viewing geometry of the optics with respect to the neutral beam. The precise alignment of the polarization filters is important as they transmit only the $\sigma$ or the $\pi$ or both components, respectively. The optical heads were adjusted with respect to each other and the base plate (see Figure 1(b)) using a mock-up of the beam geometry in the lab. In order to align the polarizers, a reference had to be established for the beam mock-up. For this purpose, a reference polarizer was installed into a motorized rotation stage which allows us to turn the reference polarizer by $360^{\circ}$. Measuring the signal variation of an integrating sphere by rotating the reference polarizer, the minimum and maximum can be determined and thus the polarizer of each optical head can be adjusted.

An in-vessel radial calibration is carried out to determine the intersection points between the views and the neutral beam. Beam-into-gas discharges with and without toroidal magnetic field are carried out to perform a relative calibration of the different LOS and to absolutely calibrate the orientation direction of the polarizer.

The beam emission line profiles are characterized by forward modelling the measured spectra of beam into gas discharges without magnetic field. Without the presence of a magnetic field, there is no splitting of the $\mathrm{D}_{\alpha}$ spectral line. For each beam energy component, a narrow peak is seen in the spectrum as expected from the beam model (see Figure 3(a)). The three peaks are superposed by a wide spectral feature which is attributed to reflections inside the optical head. The line profile shape is approximated by fitting these peaks using a modified Gaussian, consisting of a Gaussian and a Lorentz profile, for each of the three beam energy components. Measurements on other tokamaks reported similar line profile shapes and were attributed to polarized background emission ${ }^{7}$ and broadening of the emission profile due to the scattering of the beam particles on the background gas. ${ }^{8}$

\section{FIRST MEASUREMENTS}

A forward model for the Stark split $\mathrm{D}_{\alpha}$ spectra has been developed in order to extract the magnetic field line angle. ${ }^{9}$
Each beam species (full, half, and third energy component) produces emission with a different Doppler shift and features a $\mathrm{D}_{\alpha}$ multiplet with nine spectral lines. In addition, the spectra are superposed by the $\mathrm{D}_{\alpha}$ radiation of the beam halo and the CII multiplet at $657.805 \mathrm{~nm}$ and $658.288 \mathrm{~nm}$. The forward model combines all three reference channels and fits the spectra for one radial measurement position simultaneously, starting from the magnetic field line angle. The fit variables in the forward model are the radiance of the total beam emission for each beam energy component, the radiance of the CII multiplet, and the far wing of the $\mathrm{D}_{\alpha}$ spectral line. Two fit parameters are included to take the stray radiation into account. The width of the Gaussian that describes the stray light is fitted for the full energy component and the ratio of the total beam emission feature to the stray light feature. Additional fit parameters are the spectral radiance of the background emission, three occupation numbers which describe the relative occupation of the states and account for the non-statistical population, an effective overlap length which describes the length of the LOS through the neutral beam and is different for each optical head, the beam voltage, and the magnetic field line angle. The radial electric field $E_{r}$ is used as an input and is evaluated from $\nabla p_{i} /\left(e n_{i}\right)$, where $p_{i}$ and $n_{i}$ are the ion pressure and ion density, based on edge charge exchange and electron density measurements. A typical fit using this forward model has 24 parameters.

Figures 3(b)-3(d) shows the spectra of the three optical heads in black measured in an H-mode discharge. The data are averaged over the time window 3.0-3.5 s. The areas highlighted in magenta, blue, and green correspond to the total radiance of the first, second, and third beam energy component. The grey and purple shaded areas represent the CII multiplet and the $\mathrm{D}_{\alpha}$ wing, respectively. The fits based on the combined forward modelling are marked in red and show good agreement with the measured spectra of all three optical heads. The resulting magnetic field line angle $\gamma$ is shown in Figure 3(e), while Figure 3(f) shows the normalized poloidal flux label $\left(\rho_{p o l}\right)$ of each LOS. The different colours represent the five radial positions. Here, the temporal evolution during the ELM cycle is shown for the time window 5.0-7.5 s. Note that a change in $\gamma$ is already observed before the plasma starts to move due to the ELM onset (see Figure 3(f)), showing the sensitivity of the diagnostic to the ELMs. Comparison 
to equilibrium reconstructions using the new system will be reported in the future.

\section{ACKNOWLEDGMENTS}

The authors would like to thank W. Zeidner, M. Rolffs, H. Kötterl, M. Ebner, and A. Herrmann for their support. This work has been carried out within the framework of the EUROfusion Consortium and has received funding from the Euratom research and training programme 2014-2018 under Grant Agreement No. 633053. The views and opinions expressed herein do not necessarily reflect those of the European Commission. The support from the EUROfusion
Researcher Fellowship programme under Grant No. WP14FRF-IPP/Viezzer and from the Spanish Ministry of Economy and Competitiveness (Grant No. FJCI-201422139) is gratefully acknowledged.

${ }^{1}$ P. B. Snyder et al., Phys. Plasmas 9, 2037 (2002).

${ }^{2}$ E. Viezzer et al., Nucl. Fusion 53, 053005 (2013).

${ }^{3}$ O. Marchuk et al., Plasma Phys. Controlled Fusion 54, 095010 (2012).

${ }^{4}$ E. Delabie et al., Plasma Phys. Controlled Fusion 52, 125008 (2010).

${ }^{5}$ M. F. Gu et al., J. Phys. B: At., Mol. Opt. Phys. 41, 095701 (2008).

${ }^{6}$ A. Boileau et al., J. Phys. B: At., Mol. Opt. Phys. 22, L145 (1989).

${ }^{7}$ H. Y. Yuh et al., Rev. Sci. Instrum. 79, 10F523 (2008).

${ }^{8}$ N. A. Pablant et al., Rev. Sci. Instrum. 81, 10D729 (2010).

${ }^{9} \mathrm{R}$. Dux et al., in 42nd EPS Conference, P1.121, Lisbon, 2015. 